\title{
Self-Adjustment Mechanism Guaranteeing Asymptotic Stability of Clusters Formed by Autonomous Decentralized Mechanism
}

\author{
Hiroki Takayama, Sota Hatakeyama, and Masaki Aida \\ Graduate School of System Design, Tokyo Metropolitan University, Tokyo, Japan \\ Email: hiroki.takayama.tmu@gmail.com; sota-hatakeyama@sd.tmu.ac.jp; aida@sd.tmu.ac.jp
}

\begin{abstract}
Clustering mechanisms are crucial for scalable routing in mobile ad hoc networks (MANETs). We have previously proposed an autonomous decentralized clustering mechanism that is based on differential equations. This method adopts both the diffusion effect for autonomous decentralized control, and the drift effect for balancing with the diffusion effect. In our conventional mechanism, we need to set the strength of diffusion effect stronger than the drift effect because fine tuning for balancing both effects is difficult. However, it results in a decrease of the number of clusters by the diffusion effect, and it is a problem on forming sustainable cluster structures. We need the solution of this problem for clustering in MANET. In this paper, we propose a mechanism autonomously adjusting the effects of both diffusion and drift. We show that our proposed mechanism can form sustainable cluster structures reflecting the characteristic of our conventional mechanism. Finally, we evaluate the effectiveness of the proposed mechanism for an environment in which the fusion of subnetworks occurs.
\end{abstract}

Index Terms - self-adjustment, autonomous decentralized mechanism, clustering mechanism, structure formation, mobile ad hoc network

\section{INTRODUCTION}

The autonomous decentralized mechanism in which each node can act autonomously based on local information of itself is an effective control framework for the environment in which it is difficult for us to know the state of a whole system exactly. In particular, mobile ad hoc networks (MANETs) are one of the systems in which the mechanism is effective. This is because MANETs are composed autonomously of only mobile nodes, and the topology of MANETs changes frequently with the movement of nodes [1], [2]. In MANETs, since routing schemes based on a flat structure have scalability problems, the hierarchical structure is essential to introduce dynamic routing [3]-[8]. For example, communication overhead of representative proactive protocol as routing protocol for a flat structure increases with the square of number of nodes [9].

Manuscript received October 16, 2013; revised February 4, 2014.

This work was supported by a Grant-in-Aid for Scientific Research (B) No. 23300031 (2011.2013) from the Japan Society for the Promotion of Science, and by National Institute of Information and Communications Technology (NICT).

Corresponding author email: aida@sd.tmu.ac.jp.

doi:10.12720/jcm.9.2.180-187
From the above background, we have previously proposed autonomous decentralized clustering mechanism to introduce hierarchical structures in MANETs [10]. In our mechanism, each node has values of a certain distribution in advance, and nodes exchange a portion of their value with the adjacent nodes by obeying a predefined action rule based only on local information. After that, our mechanism can form spatial structures in the network, then cluster structures are decided based on local maximum values of the distribution. We also have verified the effectiveness of our mechanism by comparing with autonomous decentralized clustering mechanism based on bio-inspired approach which is well known autonomous decentralized mechanism [11], [12]. In this verification, we showed that our mechanism can act about 30 times faster than the mechanism based on bio-inspired approach [13]. Therefore this suggests the possibility that our mechanism has the flexibility for dynamic change of network topology with movement of nodes.

In large scale MANETs, the most drastic changes of network topology are fusion and division of networks. The fusion of networks means that a node group encounters the other node groups which are outside of communication range and that these node groups enter to inside of communication range. On the other hand, the division of networks means that a node group is divided into some node groups with movement of nodes. In these drastic changes, cluster structures of different subnetworks need common criteria when fusion of subnetworks occurs. This is because cluster structures cannot be formed based on the common criteria when the fusion of different subnetworks occurs. Therefore cluster structures need to have common criteria even in different subnetworks. However, cluster structures formed by our mechanism do not have the common criterion. In our mechanism, the magnitude of the amplitude of the distribution in different subnetworks varies. Here, we define the magnitude of the amplitude of the distribution as the difference between maximum of the distribution and minimum of the distribution. If subnetworks have different amplitudes, subnetworks cannot create appropriate clustering based on the common criteria because cluster structures having the huge amplitude strongly affect clusters formation when the fusion of subnetworks occurs (Fig. 1). To avoid this problem, different subnetworks should have the common 
amplitude. The most primitive way to realize the common amplitude is to set appropriately the values of parameters in the clustering mechanism, in advance. However, [14] clarified that setting the value of parameters is difficult. Therefore, in this paper, we propose a self-adjustment mechanism that can adjust the value of parameters autonomously so as to uniform the magnitude of the amplitude.

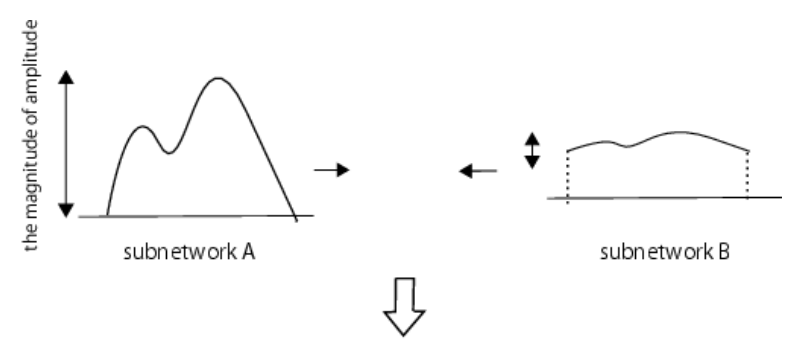

The clusters of subnetwork B are NOT maintained

Fig. 1. Fusion of subnetworks having different amplitude

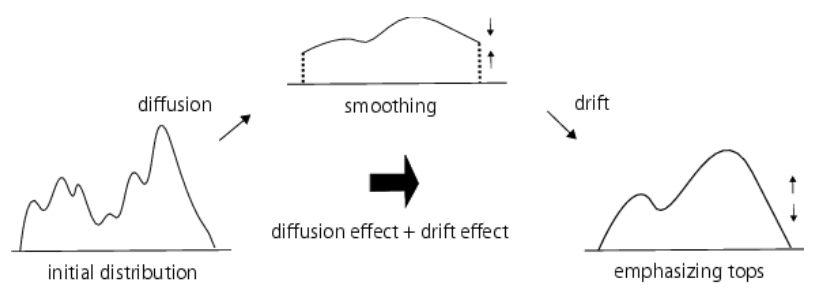

Fig. 2. Summary of our autonomous decentralized clustering mechanism

This paper is organized as follows: Section II briefly explains our autonomous decentralized clustering mechanism for introducing hierarchical structures for ad hoc networks. Section III proposes the self-adjustment mechanism that can adjust the magnitude of amplitude. Section IV investigates whether the self-adjustment mechanism can adjust the parameters so as to uniform the magnitude of the amplitude. Section $V$ verify that the selfadjustment mechanism can maintain cluster structures of subnetworks when the fusion of subnetworks occurs. In section VI, we conclude this paper.

\section{Autonomous DeCENTRALIZED CLUSTERING MECHANISM}

We briefly explain our autonomous decentralized clustering mechanism in continuous 1-dimensional space [10]. Let the density distribution of a certain quantity at position $x$ at time $\mathrm{t}$ in space be $q(x, t)$. The value of initial distribution $q(x, 0)$ is determined based on a certain metric, and each position $x$ exchanges a part of the value of $q(x, t)$ with adjacent positions by obeying predefined rule. For example, a certain metric is chosen based on battery energy or node degree. By continuing the exchange, this mechanism can form spatial structures of clusters that is described by $q(x, t)$.

Here, we explain the predefined rule for exchanging the value of $q(x, t)$. Let $J(x, t)$ be 1 -dimensional vector representing the magnitude of flow exchanging the value of $q(x, t)$ per unit of time. $J(x, t)$ is expressed by the drift term $-c f(x, t) q(x, t)$ and the diffusion term $-c \sigma^{2} \partial q(x, t) / \partial x$ as:

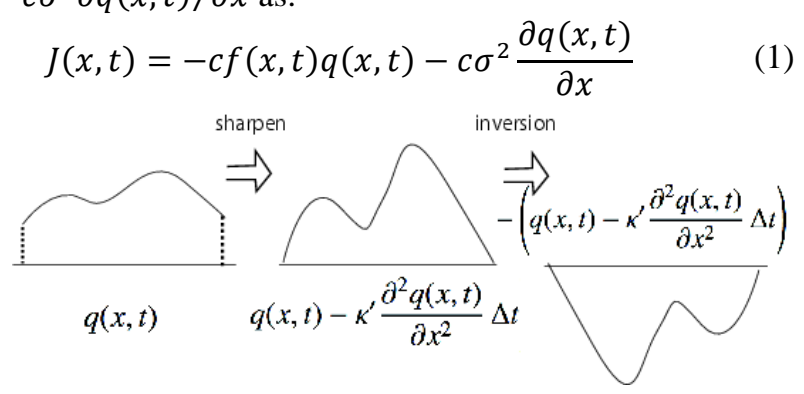

Fig. 3. Decision of potential function $\Phi(x, t)$ by using back-diffusion

where $\sigma$ and $c$ is positive constant, and $c \sigma^{2}$ needs to satisfy

$$
c \sigma^{2}<\frac{1}{2}
$$

The effect of the drift term is to emphasize the peaks of distribution, on the other hand, the effect of the diffusion term is to smooth the distribution. Our mechanism forms the spatial structures by balance between the drift effect and the diffusion effect (Fig. 2). The function $f(x, t)$ is determined by a potential function $\Phi(x, t)$ as

$$
f(x, t)=-\frac{\partial \Phi(x, t)}{\partial x}
$$

The drift effect should be opposite effect compared with the diffusion effect. Thus, we decide the potential function as (4):

$$
\Phi(x, t+\Delta t)=-\left(q(x, t)-\kappa^{\prime} \frac{\partial^{2} q(x, t)}{\partial x^{2}} \Delta t\right),
$$

where $\kappa^{\prime}$ is positive constant. Here we use $\Delta t$ as update interval to update the potential function. $-\kappa^{\prime} \partial^{2} q(x, t) / \partial x^{2} \Delta t$ in (4) means time reversal of diffusion phenomenon. That is, $-\kappa^{\prime} \partial^{2} q(x, t) / \partial x^{2} \Delta t$ in (4) sharpens the peaks of the distribution. Then, we make the distribution upside down (Fig. 3). Hereby, the drift effect can be opposite to the diffusion effect. From the above discussion, the action rule is determined by only local information of adjacent nodes. Since similar characteristic of local interaction is also true in $n$ dimensional space, this mechanism can work as a autonomous decentralized manner for arbitrary network topology.

Next, we show the action rule for an arbitrary network [10]. We define the set of nodes that are adjacent to node $i$ as $N_{i}$. We also define discretized time as $\Delta t$. The $q\left(t_{n+1}\right)$ at next time of the $q\left(t_{n}\right)$ at time $t_{n}(:=n \times \Delta t)$ of node $i$ is as following.

$$
q_{i}\left(t_{n+1}\right)=q\left(t_{n}\right)-\Delta t \sum_{j \in N_{i}}\left(J_{i, j}^{\mathrm{drift}}\left(t_{n}\right)+J_{i, j}^{\mathrm{diff}}\left(t_{n}\right)\right)
$$

where $J_{i, j}^{\text {drift }}\left(t_{n}\right)$ and $J_{i, j}^{\text {diff }}\left(t_{n}\right)$ are the variations of drift effect and diffusion effect within unit of time. Here, we define $J_{i, j}^{\text {drift }}\left(t_{n}\right)$ and $J_{i, j}^{\text {diff }}\left(t_{n}\right)$ as following. 


$$
\begin{gathered}
J_{i, j}^{\mathrm{drift}}\left(t_{n}\right):=\left\{\begin{array}{cc}
c f_{i, j} q_{i}\left(t_{n}\right), & \left(f_{i, j}\left(t_{n}\right)>0\right) \\
-c f_{i, j} q_{i}\left(t_{n}\right), & \left(f_{j, i}\left(t_{n}\right)>0\right)
\end{array}\right. \\
f_{i, j}\left(t_{n}\right):=-\left(\Phi\left(j, t_{n}\right)-\Phi\left(i, t_{n}\right)\right) \\
J_{i, j}^{\mathrm{diff}}\left(t_{n}\right):=-\sigma^{2}\left(q_{j}\left(t_{n}\right)-q_{i}\left(t_{n}\right)\right)
\end{gathered}
$$

We explain about the above equation. Because the drift effect emphasizes peaks of the distribution, a part of the distribution moves to $j$ from $i$ (to $i$ from $j$ ) in the case of $f_{i, j}\left(t_{n}\right)>0\left(f_{j, i}\left(t_{n}\right)>0\right)$. That is, a part of distribution moves to nodes having the smaller potential value from nodes having the larger potential value. Here, equation $f_{i, j}\left(t_{n}\right)=-f_{j, i}\left(t_{n}\right)$ holds. On the other hand, because the diffusion effect flats the distribution, a part of the distribution moves to $j$ from $i$ (to $i$ from $j$ ) in the case of $q_{i}\left(t_{n}\right)>q_{j}\left(t_{n}\right)\left(q_{j}\left(t_{n}\right)>q_{i}\left(t_{n}\right)\right)$.

Next, we explain how to determine the potential function $\Phi_{\mathrm{i}}\left(t_{n}\right)$. The value of the potential function $\Phi_{\mathrm{i}}\left(t_{n+1}\right)$ of node $i$ at time $t_{n+1}$ is as follows:

$$
\Phi_{i}\left(t_{n+1}\right)=-\left(q_{i}\left(t_{n}\right)-\kappa^{\prime} \Delta \sum_{j \in N_{i}}\left(J_{i, j}^{\mathrm{back}}\left(t_{n}\right)+J_{i, j}^{\mathrm{back}}\left(t_{n}\right)\right)\right)
$$

where the $J_{i, j}^{\text {back }}\left(t_{n}\right)$ is variation created by the backdiffusion of $q_{i}\left(t_{n}\right)$ to $j$ from $i$ within unit time. Here, we require attention for deciding the variation $J_{i, j}^{\text {back }}\left(t_{n}\right)$ because the variation $J_{i, j}^{\text {back }}\left(t_{n}\right)$ has possibilities to act against our purpose. For example, nodes which are not peaks of the distribution have possibilities to be emphasized as peaks of the distribution when the variation $J_{i, j}^{\text {back }}\left(t_{n}\right)$ gives values to multi-nodes. Thus we define the variation $J_{i, j}^{\text {back }}\left(t_{n}\right)$ for networks as transferring the value towards a node having largest value among adjacent nodes. From the above discussion, the variation $J_{i, j}^{\text {back }}\left(t_{n}\right)$ is given as follows:

$J_{i, j}^{\text {back }}\left(t_{n}\right)=\left\{\begin{array}{cl}q_{j}\left(t_{n}\right)-q_{i}\left(t_{n}\right) & \left(\Delta q_{i}^{\max }=q_{j}\left(t_{n}\right)-q_{i}\left(t_{n}\right)\right) \\ 0 & \text { (otherwise) }\end{array}\right.$

$$
\Delta q_{i}^{\max }\left(t_{n}\right):=\max \left(\max _{j \in N_{i}}\left(q_{j}\left(t_{n}\right)-q_{i}\left(t_{n}\right)\right), 0\right)
$$

Also, the value of parameters $c \sigma^{2}$ needs to satisfy

$$
c \sigma^{2}<\frac{1}{d_{\max }}
$$

where $d_{\max }$ is the maximum degree of nodes in the network. This is because if the value of the parameters $c \sigma^{2}$ are larger than that of (12), the values of some nodes become negative value by the diffusion effect.

Next we explain how to decide clusters. Clusters are based on local maximal value. That is, when each node tracks along the direction of the steepest ascent of the distribution, each node reaches local maximal value of the distribution. By basing on this idea, a cluster is defined by nodes reaching the same peak, and each point having maximal value is a cluster-head.

\section{SELF-ADJUSTMENT MECHANISM}

The above mentioned mechanism can form cluster structures by basing on the predefined local rule of (1). In this procedure, we need to adjust the balance between the drift effect and the diffusion effect, in advance. Here, we consider about how to set parameters of these effects (Fig. 4). If we set parameters with the drift effect stronger than the diffusion effect, some peaks are over-emphasized by the drift effect. Therefore spacial structures are not formed when we set parameters with the drift effect stronger than the diffusion effect. On the other hand, if we set parameters with the diffusion effect stronger than the drift effect, we can get spacial structures with a certain spatial size. Thus we have ever set parameters with the diffusion effect stronger than the drift effect in simulation studies. However, if we set parameters like that, the magnitude of the amplitude of the distribution decays to zero exponentially. This is because the diffusion strongly affects the amplitude of the distribution. Of course cluster structures are maintained even if time progresses because the amplitude of the distribution has no relation with cluster structures. However, when drastic change like the fusion of subnetworks occurs, they have different amplitudes, in general. This causes that cluster structures in a subnetwork having small magnitude of the amplitude cannot maintain when the fusion of different subnetworks occurs because a subnetwork having large magnitude of the amplitude has a huge effect on the cluster structures. Although one solution for the problem is to become the diffusion effect equaling to the drift effect by setting parameters, it is difficult [14].

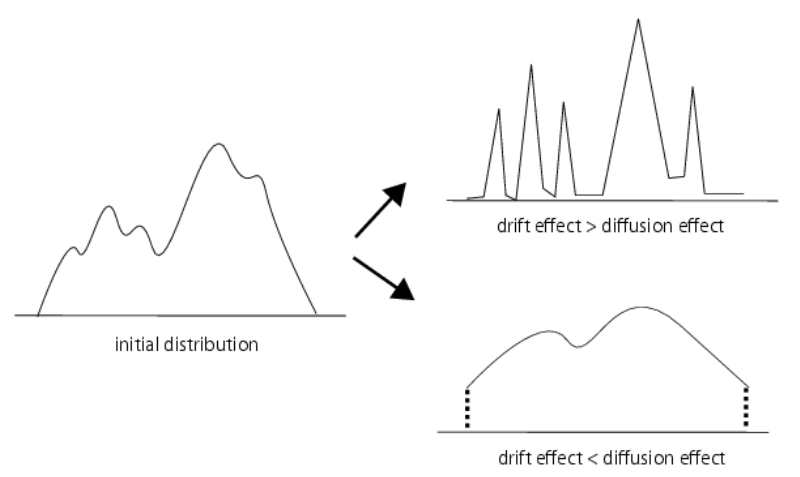

Fig. 4. Balance between drift effect and diffusion effect

We can consider two approaches to solve this problem.

One is an approach to stop the smoothing efect of the distribution. This approach is discussed in [14]. In this approach, nodes record a value of the distribution of each time, and each node uses a value of the distribution of the same time even if in different subnetworks. Thereby, this approach can uniformize the magnitude of the amplitude between different subnetworks. Another is an approach to keep a balance between drift and diffusion effects adap- 
tively, by self-adjustment. In this approach, we do not need special configuration like a memory to record the value of distributions required in a prior approach. Thus, this paper discusses the later approach.

To consider about the approach based on selfadjustment, we need to consider relative strength between the drift effect and the diffusion effect. In conventional mechanism, since the relative strength decided by setting parameters does not change even if time progresses, the amplitude of the distribution continues to decay in the case of the diffusion effect stronger than the drift effect. Hence we consider a mechanism that adjusts the relative strength autonomously even if time progresses in order to become the diffusion effect equaling to the drift effect. The concept of the mechanism is as following: If the drift effect is stronger than the diffusion effect, the mechanism changes autonomously the diffusion effect to strong effect. On the other hand, if the diffusion effect is stronger than the drift effect, the mechanism changes autonomously the diffusion effect to weak effect.

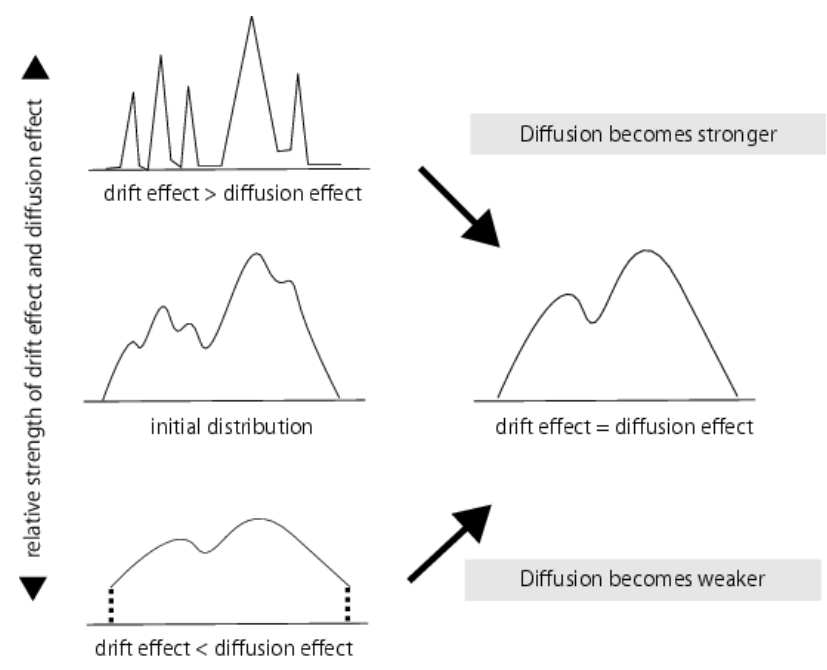

Fig. 5. Concept of self-adjustment mechanism

Thereby, the mechanism can equalize the two effects autonomously.

By basing on the above concept, we introduce a function changing the relative strength to the diffusion term of (1). Here, to introduce the function, we use the fact that the gradient of the distribution becomes likewise larger when the magnitude of the amplitude becomes larger. Also, to use the fact is suitable for autonomous decentralized control because the gradient of distribution is decided by only local information. From the above discussion, we use the function as a diffusion coefficient which depends on the absolute value of the gradient of distribution, and try to govern the strength of diffusion effect adaptively. Let us introduce the extended local action rule $J(x, t)$ as follows:

$$
J(x, t)=-c f(x, t) q(x, t)-c \sigma^{2} g(|\nabla q(x, t)|) \nabla q(x, t),
$$

where $g(|\nabla q(x, t)|)$ is a function to adjust the strength of the diffusion effect.
The actual form of the function $g(\cdot)$ should be determined according to the following three conditions.

- $g(\cdot)$ should be monotonically increasing function.

- $g(\cdot)$ should have the upper-bound.

- In order to keep the amplitude of distribution at an appropriate value, $g(\cdot)$ should have large gradient around the corresponding value of $|\nabla q(x, t)|$

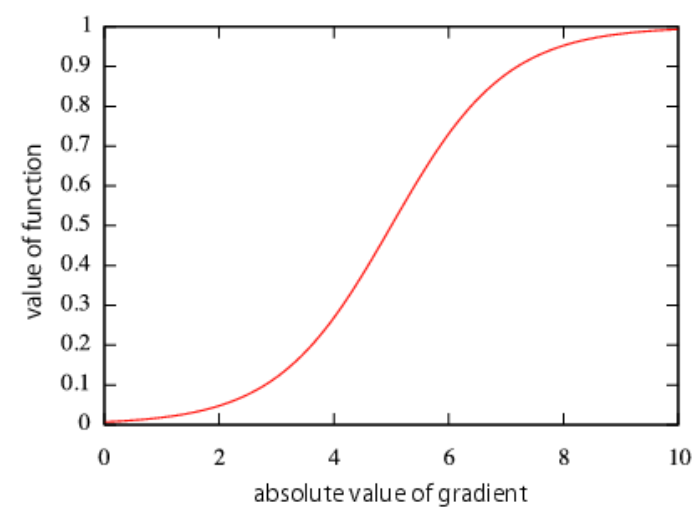

Fig. 6. Typical example of function $g(|\nabla q(x, t)|)$ satisfying the three conditions

The first condition is required for stabilizing the amplitude of the distribution. Since the larger value of $|\nabla q(x, t)|$ means stronger drift effect, stronger diffusion effect is required. Therefore, $g(|\nabla q(x, t)|)$ should become large. Conversely, for small value of $|\nabla q(x, t)|$, $g(|\nabla q(x, t)|)$ should become small. The second condition concerns the limitation (14) of the maximum value of diffusion coefficient. In the case of (13), the parameters $c \sigma^{2}$ need to satisfy

$$
g(|\nabla q(x, t)|) c \sigma^{2}<\frac{1}{d_{\max }}
$$

Then if the function does not have the upper-bound, we cannot set the value of the parameters $c \sigma^{2}$. Conversely, if $g$ has the upper-bound, we can set the parameters $c \sigma^{2}$ to satisfy the condition (14). The last condition concerns the position of the equilibrium in self-adjustment mechanism. Let us consider that appropriate magnitude of the amplitude is featured by the corresponding gradient $|\nabla q(x, t)|=a$. Then, $a$ should be the equilibrium. This means that the gradient of $g(|\nabla q(x, t)|)$ should have larger value around $|\nabla q(x, t)|=a$.

From the above discussion, we can choose

$$
g(|\nabla q(x, t)|)=\frac{1}{1+e^{-(|\nabla q(x, t)|-5.0)}}
$$

as a typical example of the function $g(|\nabla q(x, t)|)$ satisfying the above three conditions. This function is the logistic curve shown in Fig. 6. Here in Fig. 6, the vertical axis of the results expresses the value of the function $g$, and horizontal axis of the results expresses the magnitude of the amplitude. In this example, parameter $c \sigma^{2}$ need to satisfy the limitation

$$
c \sigma^{2}<\frac{1}{d_{\max }}
$$


because the upper-bound of $g$ is 1 . This is the same condition as (12) in the previous mechanism.
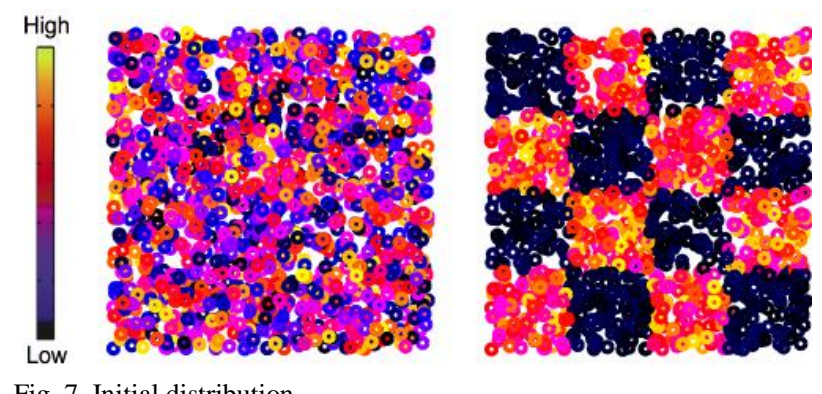

Fig. 7. Initial distribution

TABle I: PARAMETER SETTING (1)

\begin{tabular}{|c|c|c|}
\hline & randomized structure & patterned structure \\
\hline \hline$c$ & $13 \times 10^{-6}$ & $2 \times 10^{-5}$ \\
\hline$\kappa^{\prime}$ & 0.5 & 0.5 \\
\hline$c \sigma^{2}$ & 0.01 & 0.01 \\
\hline
\end{tabular}

\section{EVALUATION FOR ASYMPTOTIC STABILITY OF CLUSTERS}

In this section, we verify that our mechanism can converge the amplitude of the distribution on a finite constant value, through simulation.

\section{A. Simulation Methodology}

In this subsection, we explain simulation methodology of the verification. In this verification, we use a unit disk graph having torus boundary to exclude the influence of the boundary. We can obtain the unit disk graph through following procedure. We arrange some nodes randomly, and then we set a circle of certain length around each node. If nodes are inside the circle, we set links for these nodes. Here, since we can assume the circles as wireless communication range, the unit disk graph is one of the models which can express the topology of MANETs. Also, we set 1,600 nodes on a plane of $1 \mathrm{~km} \times 1 \mathrm{~km}$, and use $60 \mathrm{~m}$ as communication range. We also use two initial distributions as Fig. 7 in which the value of distributions is described by using color map. The left figure in Fig. 7 expresses randomized structure. Here, the value of the left figure in Fig. 7 is set to random value using the uniform distribution between $[0,10]$. The right figure in Fig. 7 expresses patterned structure. Here, the smaller valued area of the right figure in Fig. 7 is set to random value using the uniform distribution between $[0,1]$, and the larger valued area of the right figure in Fig. 7 is set to random value using the uniform distribution between $[0$, 10]. For example, we can assume the value of the randomized structure as battery energy. We also can assume the patterned structure as an environment having powersupply. We show parameters used in the evaluation in Table I.

We use two evaluation indexes. One is that the amplitude of the distribution can converge on a finite constant value from two initial conditions. The other is that the mechanism can maintain the characteristic of our conven- tional mechanism. In the former evaluation, we consider a way to evaluate as follows. First we estimate a convergence value for the time progress of the amplitude. Second we subtract the estimated value from the amplitude. Finally we plot the differences in semi-log scale, then if the differences decayed to zero exponentially, we consider the magnitude of the amplitude as converging on a finite constant value. In later evaluation, we verify that the mechanism can form cluster structures based on initial distribution by using the patterned structure. Here, if cluster structures formed by self-adjustment mechanism are same structures with cluster structures formed by our conventional mechanism, we assume our mechanism can maintain the characteristic of our conventional mechanism.

B. Evaluation

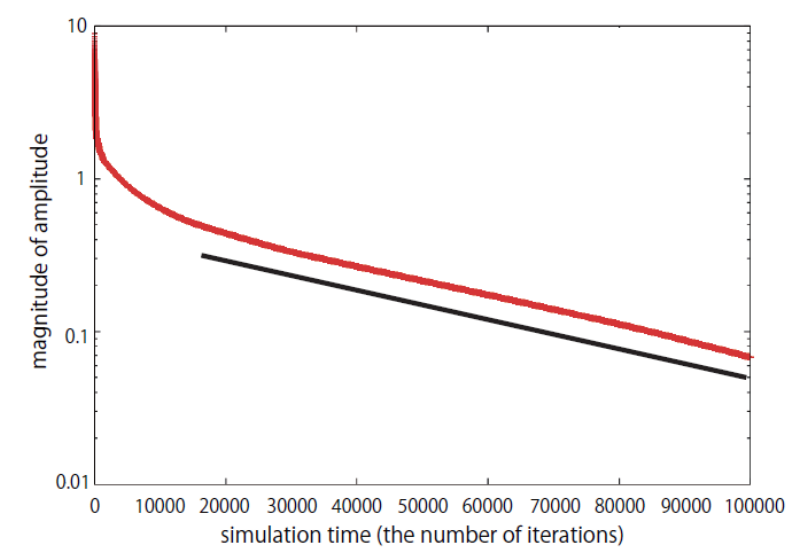

Fig. 8. Temporal variation of amplitude for randomized structure

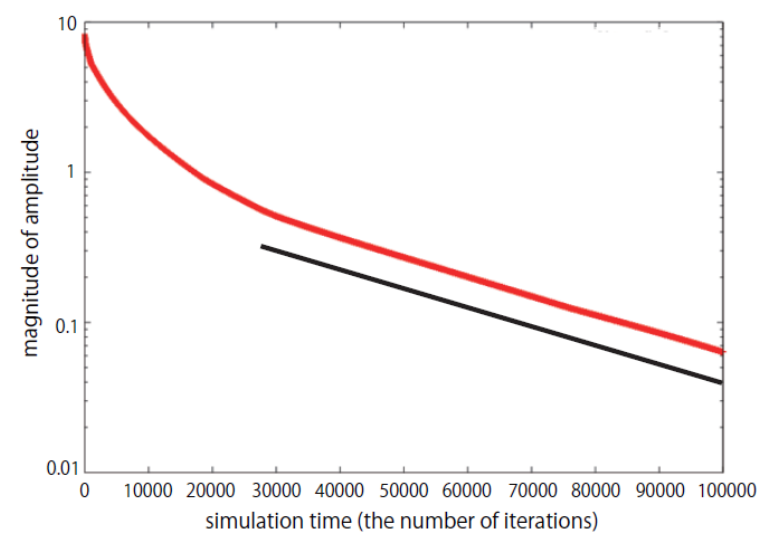

Fig. 9. Temporal variation of amplitude for patterned structure

In this subsection, first we evaluate whether the magnitude of the amplitude converges on a finite positive constant. Fig. 8 and Fig. 9 show the results in which we subtract the estimated value from time progress of amplitude and plotted in semi-log scale. Here, the vertical axis of the results expresses the magnitude of the amplitude, and horizontal axis of the results expresses the time. From the result of Fig. 8 and Fig. 9, we can see that the mechanism can converge on a finite constant. Here, we estimated the value of convergence as 0.2 in the randomized structure, we also estimated the value as 1.4 in the patterned structure. Next we verify that the advantage of our conven- 
tional mechanism is inherited to the self-adjustment mechanism. The advantage of the prior mechanism is to form cluster structures based on initial condition. Hence if cluster structures formed by our mechanism are same structures with our conventional mechanism, we can confirm that our mechanism can maintain the characteristic of our conventional mechanism. The left panels of Fig. 10 and Fig. 11 describe the value of the distribution and the right panels of the Fig. 10 and Fig. 11 describe cluster map that different clusters are denoted by different colors. Also, Fig. 10 is the result of self-adjustment mechanism. On the other hand, Fig. 11 is the result of our conventional mechanism. By comparison with cluster structures formed by prior mechanism, we can see that our mechanism has the same characteristic with prior mechanism. From the above evaluation, we verified that our mechanism can maintain the amplitude of distribution, and can maintain the characteristic of our conventional mechanism.
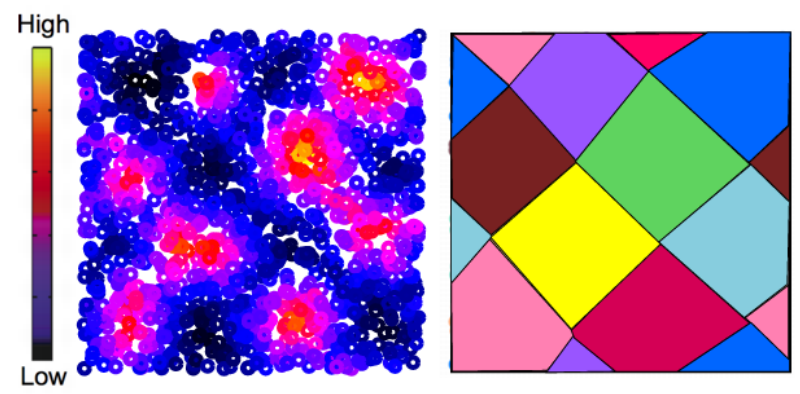

Fig. 10. Cluster structures formed by self-adjustment mechanism
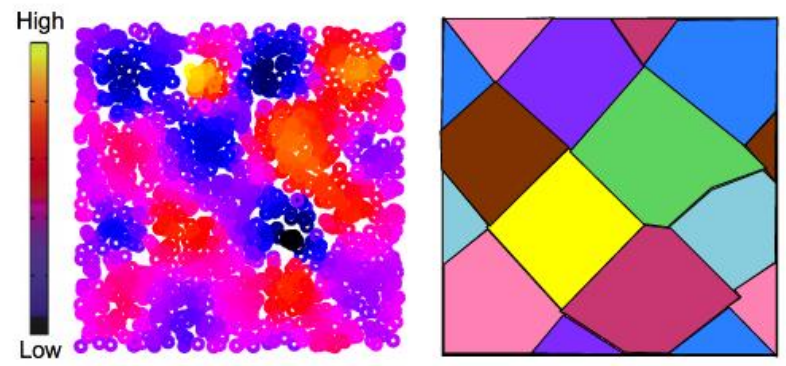

Fig. 11. Cluster structures formed by conventional mechanism

TABLE II: PARAMETER SETTING (2)

\begin{tabular}{|c|c|}
\hline parameter & value \\
\hline \hline$c$ & $2 \times 10^{-5}$ \\
\hline$\kappa^{\prime}$ & 0.5 \\
\hline$c \sigma^{2}$ & 0.01 \\
\hline
\end{tabular}

Next, we consider the different model for comparison. Let us consider the case that a function $g$ does not satisfy the abovementioned conditions. As an example of the cases, we set

$$
g(|\nabla q(x, t)|)=|\nabla q(x, t)| .
$$

We show parameters used in the evaluation in TABLE II. Here, since $g$ is an unbound function, parameter $c \sigma^{2}$ is strongly restricted. Also the function does not have large gradient around the corresponding value of $|\nabla q(x, t)|$. Hereby, we can consider that drift effect and diffusion effect cannot balance because the function does not have large gradient. From the result of Fig. 12 that describes cluster structures, we can see that cluster structures do not depend properly on characteristic in networks' initial conditions as compared to result of the prior mechanism in Fig. 11. Thus we can expect that the conditions are needed in order to achieve the self-adjustment mechanism.
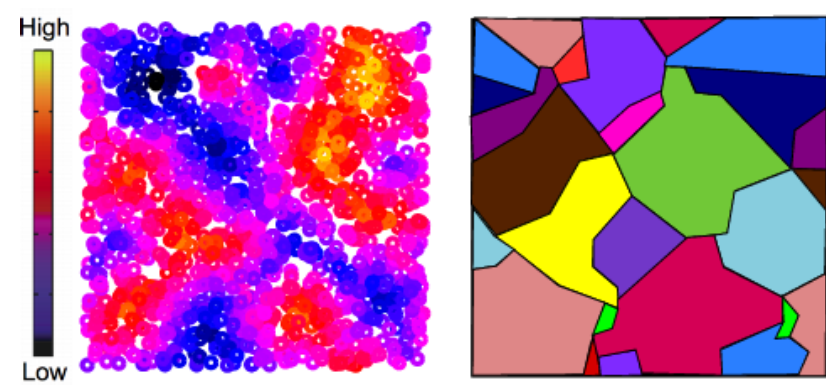

Fig. 12. Cluster structures formed in the case of different function

\section{EVALIATION FOR THE FUSION OF SUBNETWORKS}

In this section, we verify that our mechanism can maintain cluster structures based on initial distribution even if the fusion of subnetworks occurs.

\section{A. Simulation Methodology}

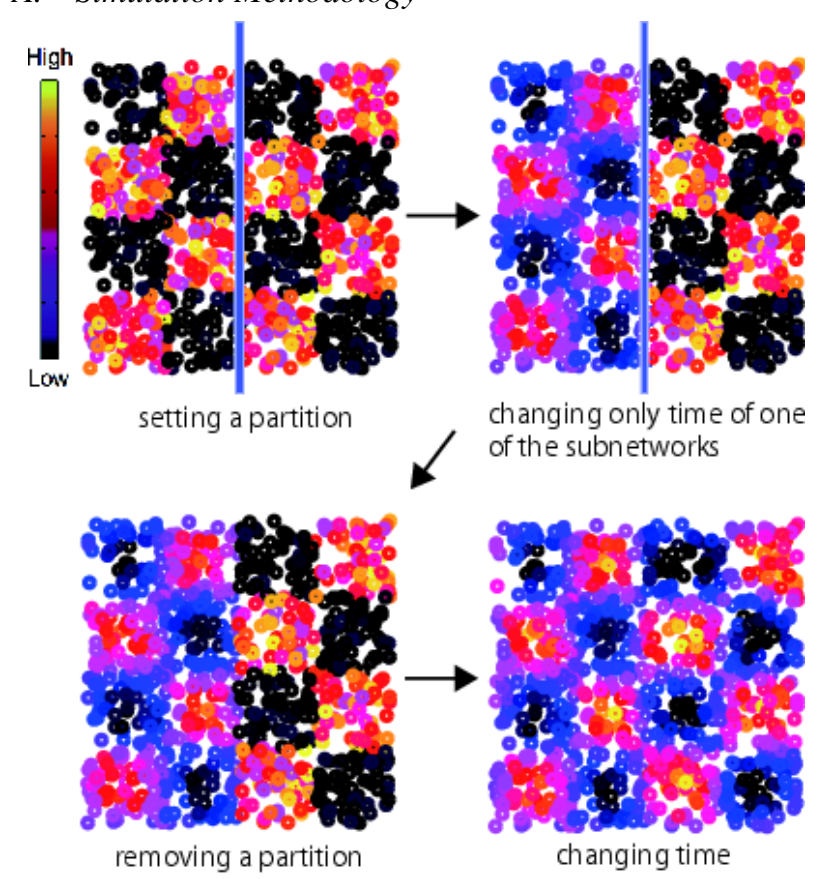

Fig. 13. Summary of the simulation model

To assume the environment in which the fusion of different subnetworks occurs, we consider a model as following in our simulation (Fig. 13). First we use the unit disk graph as network topology, and we set a partition which can interrupt communication as Fig. 13 in order to divide a network into two subnetworks. Next we change only the time of one of their subnetworks, and then we remove the partition. Here if we use the conventional mechanism, the amplitudes of subnetworks exhibit differ- 
ent behaviors. Finally we change time, that is, we create a fusion of subnetworks. Then we verify which mechanism can effectively maintain cluster structures based on initial distribution.

\begin{tabular}{|c|c|c|}
\multicolumn{3}{|c}{ TABLE III: PARAMETER SETTING (3) } \\
\begin{tabular}{|c|c|c|}
\hline & conventional mechanism & proposed mechanism \\
\hline \hline$c$ & $10^{-5}$ & $2 \times 10^{-5}$ \\
\hline$\kappa^{\prime}$ & 0.5 & 0.5 \\
\hline$c \sigma^{2}$ & 0.001 & 0.01 \\
\hline
\end{tabular}
\end{tabular}

In this verification, we use a unit disk graph having torus boundary to exclude the influence of the boundary. We set 1,600 nodes on a plane of $1 \mathrm{~km} \times 1 \mathrm{~km}$, and use $60 \mathrm{~m}$ as communication range. We remove a partition at time 1,000, and then change the time till 2,000. As initial condition, we use the patterned structure in Fig. 7. We use this initial condition as the index of this evaluation. This is because we can verify easily whether cluster structures are based on initial condition even if the fusion of subnetworks occurs.

\section{B. Evaluation}

Before the verification of our mechanism, we need to verify that the conventional mechanism cannot maintain cluster structures based on initial distribution when the fusion of subnetworks occurs. From the result of left panel in Fig. 14, we can confirm that the magnitude of the amplitude between subnetworks varies. From the result of right panel in Fig. 14, we can also confirm that cluster structures are not based on initial distribution. This is because cluster structures having the huge amplitude strongly affect clusters formation. From the results, we confirmed that cluster structures formed by prior mechanism are not formed based on initial distribution when the fusion of subnetworks occurs.

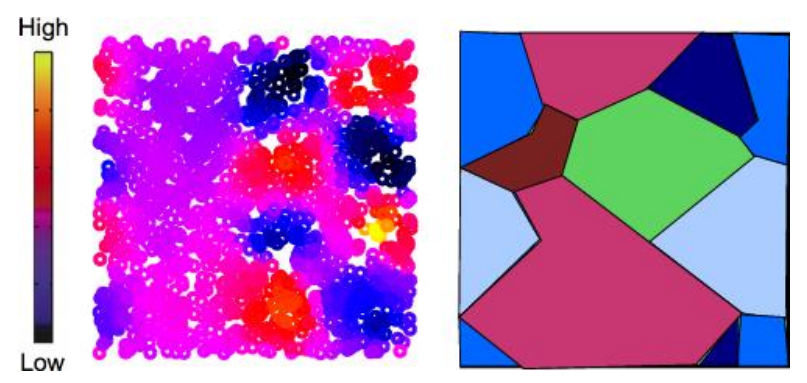

Fig. 14. Fusion of subnetworks in the case of conventional mechanism

Next we verify the effectiveness of our mechanism. rom the result of left panel in Fig. 15, we can recognize that the magnitude of amplitude between subnetworks varies but it is smaller than the result of Fig .14. Also, from the result of right panel in Fig. 15, we can recognize that our mechanism can form cluster structures based on initial distribution by comparison to cluster structures formed by the conventional mechanism in Fig. 11. From the results, we confirmed that our mechanism can form cluster structures based on initial condition even if the fusion of subnetworks occurred.
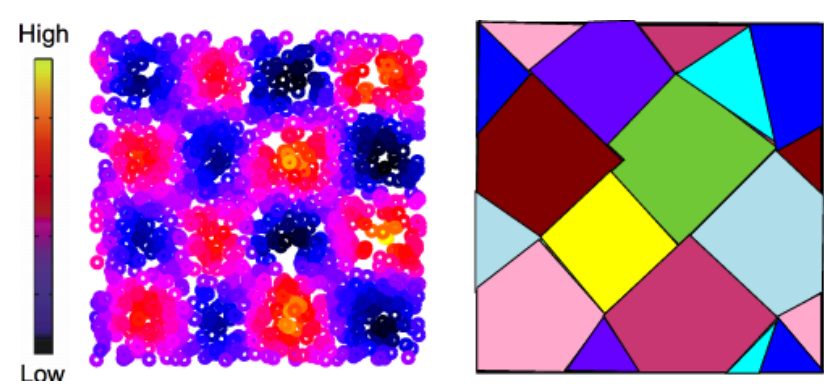

Fig. 15. Fusion of subnetworks in the case of self-adjustment mechanism

\section{CONCLUSIONS}

In this paper, we proposed the self-adjustment mechanism that adjusts the magnitude of the amplitude to become the finite magnitude. In order to evaluate the effectiveness of the self-adjustment mechanism, we verified whether the magnitude of amplitude converges on a finite positive constant by using two initial conditions that are important metrics ensuring the effectiveness of the mechanism. We also verified whether our mechanism can form cluster structures reflecting the characteristic of our conventional mechanism. Finally we evaluated the effectiveness of self-adjustment mechanism for an environment in which the fusion of subnetworks occurs. Future studies will investigate for topology change with movement of nodes.

\section{ACKNOWLEDGMENT}

This work was supported by a Grant-in-Aid for Scientific Research (B) No. 23300031 (2011-2013) from the Japan Society for the Promotion of Science, and by $\mathrm{Na}$ tional Institute of Information and Communications Technology (NICT).

\section{REFERENCES}

[1] J. Wu, I. Stojmenovic, “Ad hoc networks," IEEE Computer, vol. 37, no. 2, pp. 29-31, 2004.

[2] C. E. Perkins, "Ad hoc networking," Addison-Wesley, 2000.

[3] A. B. McDonald and T. F. Znati, "A mobility-based frame work for adaptive clustering in wireless ad hoc networks," IEEE JSAC, vol. 17, pp. 1466-87, 1999.

[4] C. R. Lin and M. Gerla, "Adaptive clustering for mobile wireless networks,” IEEE JSAC, vol. 15, pp. 1265-75, 1997.

[5] T. C. Hou and T. J. Tsai, "An access-based clustering protocol for multihop wireless ad hoc networks," IEEE JSAC, vol. 19, no. 7, pp. 1201-10, 2001.

[6] C. R. Lin and M. Gerla, "A distributed control scheme in multihop packet radio networks for voice/data traffic support," in Proc. IEEE ICC'95, vol. 2, 1995, pp. 1238-42.

[7] U. C. Kozat, G. Kondylis, B. Ryu, and M. K. Marina, "Virtual dynamic backbone for mobile ad hoc networks," in Proc. IEEE ICC'01, vol. 1, 2001, pp. 250-55.

[8] M. R. Pearlman and Z. J. Haas, "Determining the optimal configuration for the zone routing protocol," IEEE JSAC, vol. 17, pp. 1395-414, 1999.

[9] E. M. Belding-Royer, "Hierarchical routing in ad hoc mobile networks," Wireless Communications and Mobile Computing, vol. 2, no. 5, pp. 515-532, 2002. 
[10] C. Takano, M. Aida, M. Murata, and M. Imase, "Proposal for autonomous decentralized structure formation based on local interaction and back-diffusion potential," IEICE Transactions on Communications, vol. E95-B, no. 5, pp. 1529-1538, 2012.

[11] G. Neglia and G. Reina, "Evaluating activator-inhibitor mechanisms for sensors coordination," 2nd IEEE/ACM BIONETICS, Budapest, Hungary, 2007.

[12] K. Hyodo, N. Qakamiya, E. Nakaguchi, M. Murata, Y. Kubo, and K. Yanagihara, "Reaction-diffusion based autonomous control of wereless sensor networks," Int. J. Aensor Networks, vol. 7, no. 4, pp. 189-198, 2010.

[13] K. Takagi, Y. Sakumoto, C. Takano, and M. Aida, "On convergence rate of autonomous decentralized structure formation technology for clustering in ad hoc networks," in Proc. ICDCS Workshops, 2012.

[14] R. Hamamoto, C. Takano, M. Aida, and K. Ishida, "Guaranteeing asymptotic stability of clustering by autonomous decentralized structure formation," in Proc. 9th IEEE International Conference on Autonomic and Trusted Computing, 2012.

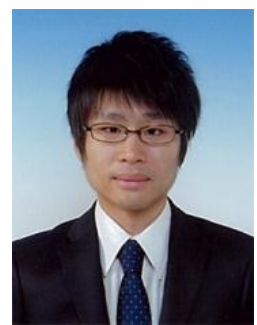

Hiroki Takayama received the B.S. degree from Tokyo Metropolitan University, Japan, in 2011. He is currently a graduate student toward the M.E. degree at the Graduate School of the System Design, Tokyo Metropolitan University. His primary interests include ad hoc networks.

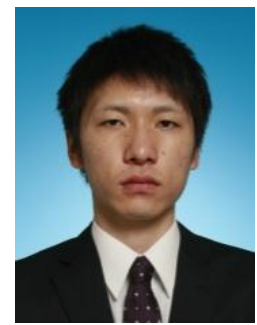

Sota Hatakeyama received the B.E. degree from Tokyo Metropolitan University, Japan, in 2012. He is currently a graduate student toward the M.E. degree at the Graduate School of System Design, Tokyo Metropolitan University. His research interests are in traffic control in information networks.

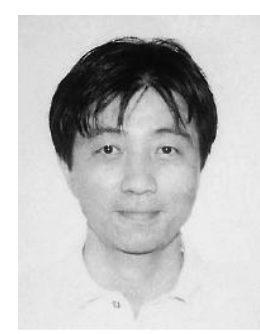

Masaki Aida received his B.S. and M.S. degrees in Theoretical Physics from St. Paul's University, Tokyo, Japan, in 1987 and 1989, respectively, and received the Ph.D. in Telecommunications Engineering from the University of Tokyo, Japan, in 1999. In April 1989, he joined NTT Laboratories. From April 2005to March 2007, he was an Associate Professor at the Faculty of System Design, Tokyo Metropolitan University. He has been a Professor of the Graduate School of System Design, Tokyo Metropolitan University since April 2007. He received the Best Tutorial Paper Award from the IEICE Communications Society in 2013. His current interests include traffic issues in computer communication networks. He is a member of the IEEE, IEICE and the Operations Research Society of Japan. 